\title{
Enhanced Mobile Node Tracking With Received Signal Strength in Wireless Sensor Networks
}

\author{
Ahmed Abdullah ${ }^{1}$, Vinolee $\mathrm{R}^{2}$ \\ ${ }^{1}$ (Department of Electronics and communication, SRM University, India) \\ ${ }^{2}$ (Department of Electronics and communication, SRM University, India)
}

\begin{abstract}
Node localization is important parameter in WSN. Node localization is required to report origin of events which makes it one of the important challenges in WSN. Received signal strength (RSS) is used to calculate distance between mobile node and reference node. The position of the mobile node is calculated using multilateration algorithm (MA). Extended Kalman filter (EKF) is utilized to estimate the actual position. In this paper, the implementation and enhancement of a tracking system based on RSS indicator with the aid of an Extended Kalman Filter (EKF) is described and an adaptive filter is derived.
\end{abstract}

Keywords - Extended Kalman filter (EKF), mobile node tracking, multilateration algorithm (MA), received signal strength (RSS), Wireless sensor networks (WSN)

\section{INTRODUCTION}

Advances in Integrated circuit technology have enabled the integration of computation and communication into one device which monitor or control physical processes. Wireless sensor network have attracted a lot of research in the recent past. The various issues related to wireless sensor networks pose interesting challenges to researcher in domain like localization. There are various location estimation methods. Received signal strength (RSS) based ranging is an advantage as it already have radios, requires no additional hardware support. Other ranging methods used are angle-of- arrival (AOA), time-of-arrival (TOA), and timedifference-of-arrival (TDOA).

RSS-based methods suffer from relatively lower accuracy. Several methods have been introduced to provide better accuracy. Kalman filter is used to improve the accuracy in tracking. In [1] tracking of mobile node is done using Kalman filter. This algorithm uses lateralization with nearest three estimated nodes to calculate the coordinates and it is implemented for straight and circular movements of the mobile node. In another algorithm [2] also used Kalman filter and proposed a novel filter base on fading Kalman filter. In [3] considered shadowing effect, a novel method by combining Kalman filter and maximum-likelihood is given.

The rest of this paper is organized as follows: The system model, the wireless channel propagation model, the multilateration algorithm are described in section II. In section III, Extended Kalman filter model is presented. In section IV, the simulation results are given and the performance is analyzed with comparison of different methods. Finally, conclusions are given in section V.

\subsection{System model}

\section{SYSTEM MODEL}

A system model as given in literature [2] is used which has an area $20 \mathrm{~m} \times 20 \mathrm{~m}$. The mobile node does uniform motion in this area and its direction may change at any time. Four reference nodes are distributed at four corners of this area. The reference nodes sends wireless signal at same time and for every time period T. The computer simulations are performed on a special trajectory which is a circle starting from $(14,8)$ and whose center is $(8,8)$ and radius is 6 as shown in the Fig 1.

\subsection{Wireless channel propagation model}

The scattering, reflection and blockage of physical obstacles results in random variations of the signal which should be statistically modelled. And the most common model which is empirically confirmed is lognormal shadowing. This model is presented in [3] as following equation:

$$
P_{r}=P_{o}-10 \gamma \log _{10}\left(\frac{d}{d_{o}}\right)+X_{\sigma}
$$

Where $P_{r}$ is the received signal strength (RSS) of the receiver which is d away from the transmitter. Similarly, $P_{o}$ is the received signal strength of the receiver which is $d_{o}$ away from the transmitter. $\gamma$ is path loss exponent, which generally equals from 2 to 4 . $\mathrm{d}$ denotes the distance between the transmitter and the receiver 
and $d_{0}$ denotes the reference distance. X $\sigma$ denotes the Gaussian random noise with mean value of zero and variance of $\sigma^{2}$.

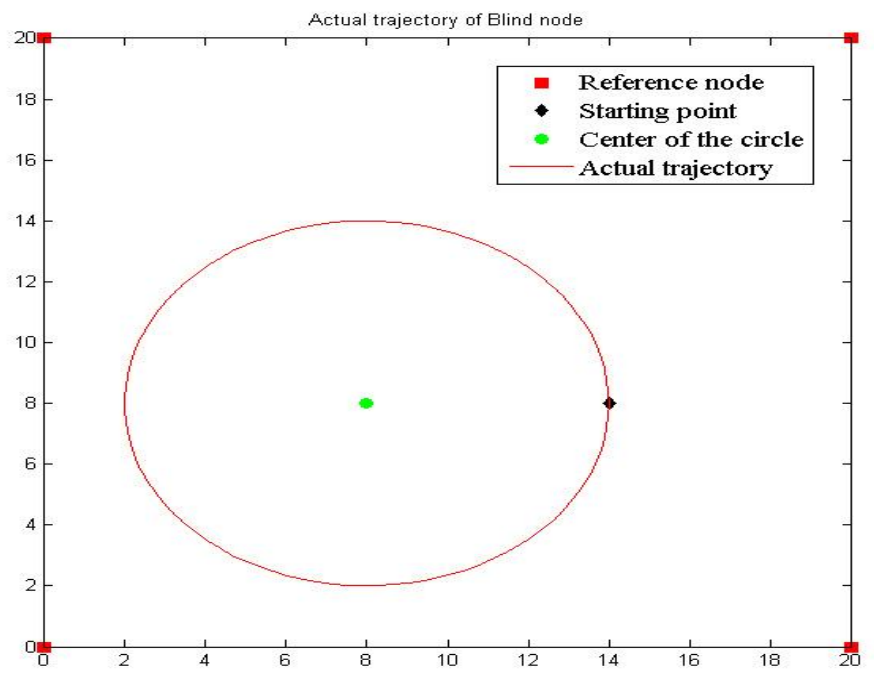

Fig. 1. Actual trajectory of mobile node.

\subsection{Multilateration algorithm}

The distance between the mobile node and the reference nodes are calculated from equation (1) and the coordinates of the reference nodes are known, multilateration algorithm (MA) can be used to estimates the coordinates of mobile node. The multilateration algorithm is an effective location algorithm.

Let the coordinates of the ith reference node be $\left(\mathrm{x}_{\mathrm{i}}, \mathrm{y}_{\mathrm{i}}\right)$, and the distance between the mobile node and the ith reference node be $\mathrm{d}_{\mathrm{i}}$. The minimum mean square error matrix [4] of the coordinates of the mobile node can be given by:

$$
\begin{gathered}
\stackrel{\Lambda}{X}=\left(A^{T} A\right)^{-1} A^{T} b \\
\text { where } \stackrel{\Lambda}{X}=\left[\begin{array}{c}
\Lambda \\
x \\
\Lambda \\
y
\end{array}\right] ; A=\left[\begin{array}{cc}
2\left(x_{1}-x_{n}\right) & 2\left(y_{1}-y_{n}\right) \\
2\left(x_{2}-x_{n}\right) & 2\left(y_{2}-y_{n}\right) \\
2\left(x_{3}-x_{n}\right) & 2\left(y_{3}-y_{n}\right) \\
\cdot & \cdot \\
\cdot & \cdot \\
2\left(x_{n-1}-x_{n}\right) & 2\left(y_{n-1}-y_{n}\right)
\end{array}\right] \& \\
b=\left[\begin{array}{c}
x_{1}^{2}-x_{n}^{2}+y_{1}^{2}-y_{n}^{2}-d_{1}^{2}+d_{n}^{2} \\
x_{2}^{2}-x_{n}^{2}+y_{2}^{2}-y_{n}^{2}-d_{2}^{2}+d_{n}^{2} \\
x_{3}^{2}-x_{n}^{2}+y_{3}^{2}-y_{n}^{2}-d_{3}^{2}+d_{n}^{2} \\
\cdot \\
\cdot \\
x_{n-1}^{2}-x_{n}^{2}+y_{n-1}^{2}-y_{n}^{2}-d_{n-1}^{2}+d_{n}^{2}
\end{array}\right] .
\end{gathered}
$$

\subsection{Extended Kalman filter algorithm}

\section{III}

\section{TRACKING}

The motion model of the mobile node has been given in [2], which presents that the mobile node does uniform linear motion in duration and its direction may change at any time.

Assuming the actual coordinates of the mobile node at time $\mathrm{k}^{\text {th }}$ is $\left(\mathrm{x}_{\mathrm{k}}, \mathrm{y}_{\mathrm{k}}\right)$, and the corresponding speed is $\left(\mathrm{V}_{\mathrm{x}}, \mathrm{V}_{\mathrm{y}}\right)$. Let the state vector of mobile node at time $\mathrm{k}^{\text {th }}$ be $S_{k}=\left(x_{k}, y_{k}, V_{x}, V_{y}\right)^{T}$, we can obtain the state equation: 


$$
\hat{\boldsymbol{S}}_{k}(-)=\left[\begin{array}{cccc}
1 & T & 0 & 0 \\
0 & 1 & 0 & 0 \\
0 & 0 & 1 & T \\
0 & 0 & 0 & 1
\end{array}\right] \cdot\left[\begin{array}{c}
x_{k-1} \\
V_{x} \\
y_{k-1} \\
V_{y}
\end{array}\right]=\phi_{k} \cdot \hat{S}_{k-1}(+)
$$

In above equation, T is the sampling period of RSS, and $\Phi_{\mathrm{k}}$ is the one step state transition matrix.

The filtering process [5] is as follows:

The initial state of filtering:

Prediction stage:

$$
\hat{S}_{o}(+)=\mu_{s o}, M_{k-1}(+)=C_{s o}
$$

$$
\hat{\boldsymbol{S}}_{k}(-)=\phi_{k} \cdot \hat{\boldsymbol{S}}_{k-1}(+)
$$

One step prediction mean square error matrix:

$$
M_{k}(-)=\phi_{k} M_{k-1}(+) \phi_{k}^{T}+Q
$$

$\mathrm{Q} \rightarrow$ covariance of process noise.

Update stage:

Filtering gain matrix:

$$
K=M_{k}(-) H_{k}^{T}\left(H_{k} M_{k}(-) H_{k}^{T}+R\right)^{-1}
$$

Filtering mean square error matrix:

$$
M_{k}(+)=\left(I-K H_{k}\right) M_{k}(-)
$$

State filtering:

Where:

$$
\hat{\boldsymbol{S}}_{k}(+)=\hat{\boldsymbol{S}_{k}}(-)+K\left(Z_{k}-H_{k} \hat{\boldsymbol{S}}_{k}(-)\right)
$$

$$
H_{k}=\left[h_{i j}^{k}\right]_{4 \times 4} \quad \mid \begin{aligned}
& h_{i, 1}^{k}=2\left(\hat{x}^{k}-x_{i}\right) \\
& h_{i, 3}^{k}=2\left(\hat{y}^{k}-y_{i}\right) \\
& h_{i, 2}^{k}=h_{i, 4}^{k}=0
\end{aligned}
$$

is the linearization of the measurement around the previous state. $\mu_{\mathrm{so}}$ is the mean value of the state vector at the initial time. Cso is the covariance matrix of the state vector at the initial time. $\mathrm{R}$ is the covariance matrix of the observation noise vector at $n_{k}$ time $k^{\text {th }}$. $I$ is the unit matrix. $R_{k}$ and $Q$ are the measurement and process noise covariance matrices respectively. The measurement noise covariance matrix is in the form of:

$$
R_{k}=\sigma_{n}^{2} \cdot I_{4}
$$

\section{ENHANCED EXTENDED KALMAN FILTER}

A significant enhancement is derived by incorporating the following adaptive modifications into the modelled EKF for object tracking.

4.1 Linearization.

The most important part of applied enhancements in the proposed method is the model presented for measurements. It is clear that the relation of RSS indicators and coordinates of the source node is nonlinear. Thus, to use EKF for estimation, a linearization of measurement is required. Consider the measurements are selected to be the same as RSS indicators. The corresponding linearization cause to the following matrices:

$$
H_{k}=\left[h_{i j}^{k}\right]_{4 \times 4} \quad \mid \begin{gathered}
h_{i, 1}^{k}=\frac{-20 \gamma\left(\hat{x}^{k}-x_{i}\right)}{\log _{10}\left(d_{k}\right)} \\
h_{i, 3}^{k}=\frac{-20 \gamma\left(\hat{y}^{k}-y_{i}\right)}{\log _{10}\left(d_{k}\right)} \\
h_{i, 2}^{k}=h_{i, 4}^{k}=0
\end{gathered}
$$


The magnitudes of elements of $H_{k}$ matrix are so much smaller than the case of Eq. 12. The importance of this point becomes clear when $\mathrm{KF}$ begins to diverge.

\section{V}

Simulation ResUlts

In this chapter, the simulation result of Extended Kalman filter (KF), Enhanced Extended Kalman filter are presented. In order to verify the effect of the Extended Kalman filter (EKF) algorithm in location and tracking, MATLAB is used to do the simulation.

As shown in figure 1, the actual trajectory of the mobile node is a circle. The starting coordinates of the mobile node is $(14,8)$, whose centre is at $(8,8)$, radius is $6 \mathrm{~m}$ and its speed is always $1 \mathrm{~m} / \mathrm{s}$. The sampling period of RSS (T) is 1 second. defined as:

To compare the performance of each method, Root Mean Square Error (RMSE) of each method is

$$
R M S E=\sqrt{\frac{1}{M} \sum_{k=1}^{M}\left[\left(x_{k}-\widehat{x}_{k}\right)^{2}+\left(y_{k}-\widehat{y}_{k}\right)^{2}\right]}
$$

Where $\left(x_{k}, y_{k}\right)$ is the actual coordinates of the blind node, and $\left(\hat{x}_{k}, \widehat{y}_{k}\right)$ is its estimated coordinates.

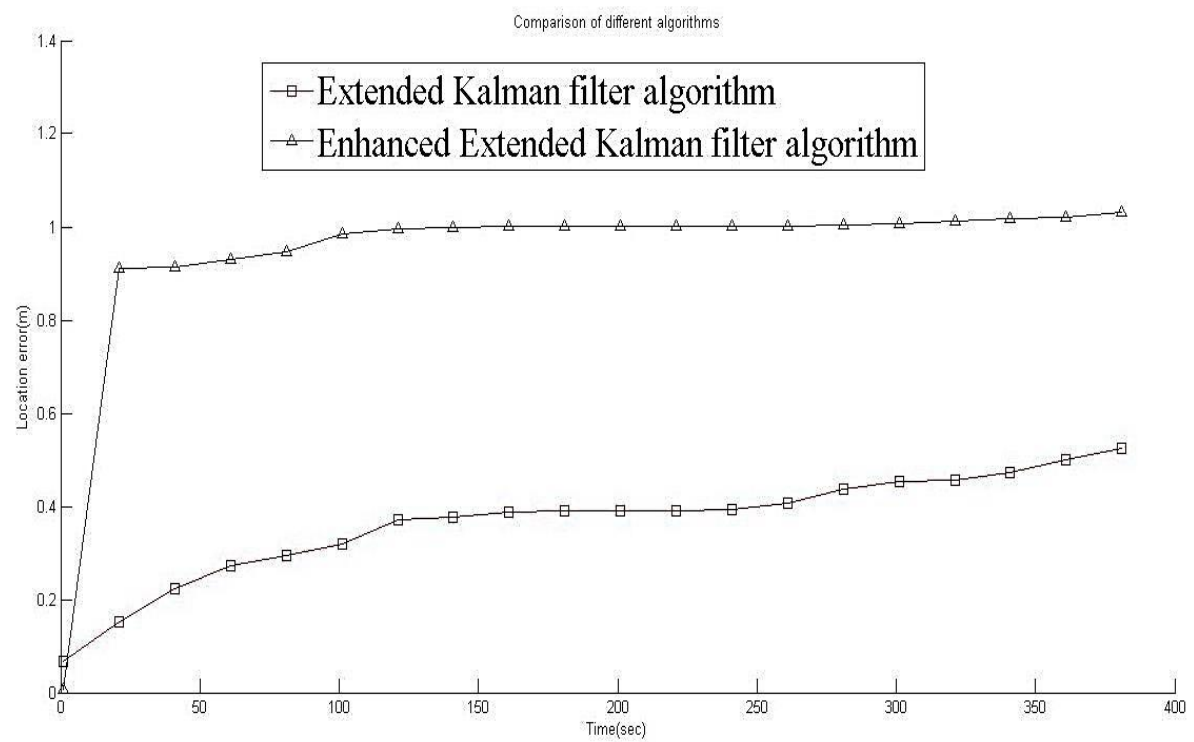

Fig. 2. Actual trajectory and estimated trajectory of mobile node.

As shown in figure 3, compared with other algorithms, the location error of Enhanced EKF algorithm is the least when compared with EKF, Kalman filter (KF), Fading Kalman filter (FKF) as presented in [2].

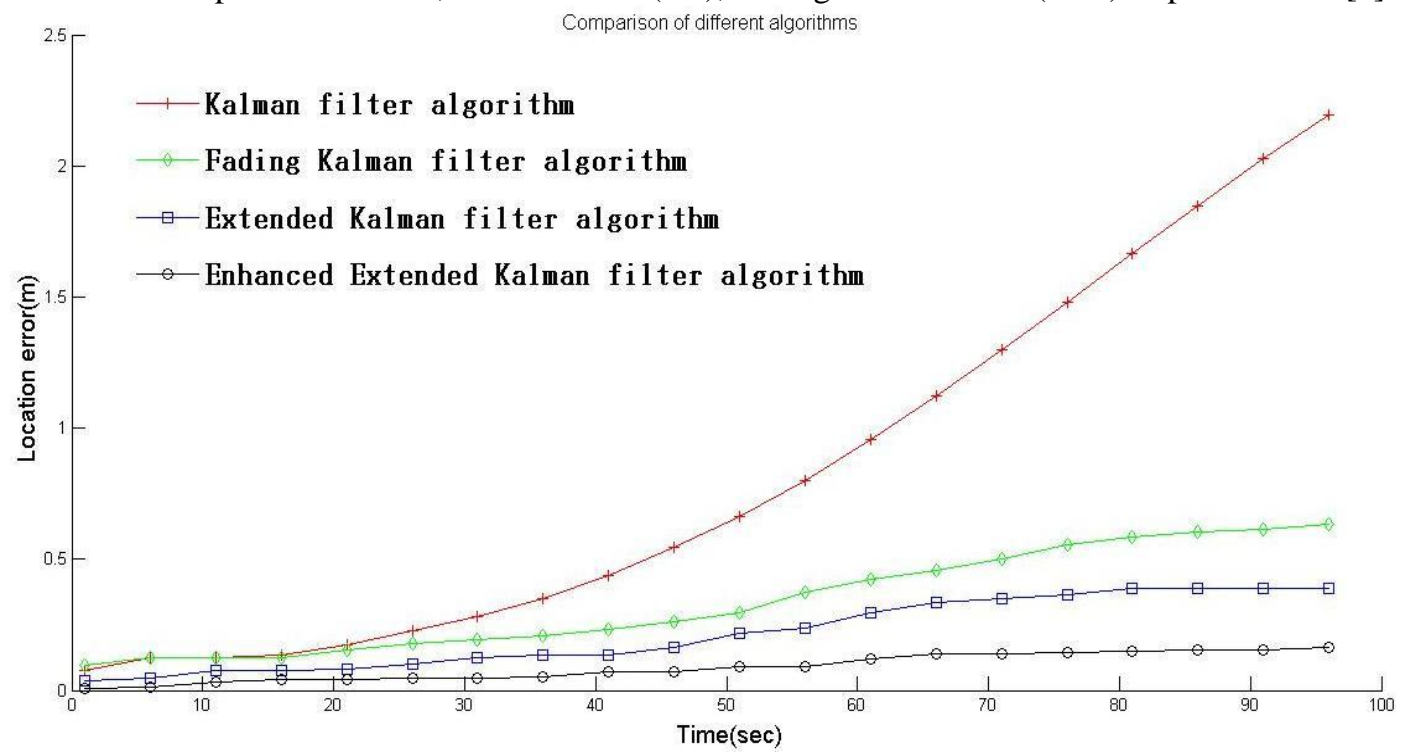

Fig. 3. Comparison of different algorithms 


\section{CONCLUSIONS}

Previous works have considered Kalman filter of single mobile nodes to smooth out the estimated trajectory. In this paper an Enhanced Extended Kalman filter is used for tracking movements of a single mobile node. RSS measurements are such modelled in EKF equations to obtain reliable solutions in tricky situations when the mobile node experiences sudden changes in its trajectory.

As compared to previously proposed methods the proposed scheme has proved to have better location accuracy in terms of the RMSE of the estimated location and faster convergence speed as compared to other models of KF.

\section{REFERENCES}

[1] S. Umesh Babu, C. S. Kumar, R. V. Raja Kumar, "Sensor Networks for Tracking a Moving Object IEEE International Conference on Industrial Technology (ICIT), Mumbai,

15-17 Dec. 2006

[2] Jieyang Yi, Liang Zhou, "Enhanced Location Algorithm with Received-Signal-Strength Using Fading Sensor Networks". IEEE Conference Publishing.2011

[3] Andrea Goldsmith. "Wireless Communications". New York: Cambridge University Press, 2005.

[4] Xiao-wei Li, Yong-jun Xu, Feng-yuan Ren. Technology of Wireless Sensor Networks. Beijing: Press Technology, 2009.

[5] Greg Welch and Gary Bishop. “An Introduction to the Kalman Filter”. Department of Computer North Carolina at Chapel Hill, 2006. using Kalman Filtering",

Kalman Filter in Wireless

of Beijing Institute of

Science University of 